\title{
Die Syphilis des Gehirns und ihre Beziehung zu anderen Erkrankungen des Nervensystems.
}

\author{
Vortrag, gehalten auf dem Congress russischer Aeryte in Moskau \\ am 5./17. Jänner 1891. \\ Von
}

\section{Dr. B. Tarnows y,}

profissor an der medicinisehen Akademie zu nit. I'otershurg.

Meine Herren! Dor Gegenstand, an don ich mir erlaube Thre Aufmerksamkeit zu fesseln, ist im Grunde genommen so umfangreich, dass er oigontlich in speciollen Tractaten und Monographion belandelt werden müsste. Doch möchte ich glauben, dass as in dieser Vorsanmmlung von Fibehgenossen, vor oinem mit der Sache gut bekitnnten Auditorium möglich sei, anch in der kurzen, für Vorträgo auf dem Congrosse bemossenen Zoit, oinige Gedanken über dieso höchst interessante Zoitfrago auszusprochen, ohne in Details einzugehen.

Dennoch abor müchle ich dio goehrte Versimmlung im Voraus um kintwchuldigung bitten, wenn sich meine liede einige Minuten über dio lestgesetzte Zeit hinaus erstrocken sollte.

Dio wissenschaltliche Behandlung der Frage über die Hirnsyphilis gehört ganz und gar unserem Jahrhundert an.

Wom sich anch oinzolue höchst soltone fiallo von Hirnsyphilis von Autoren des vorigen Jihlıhunderts in Vorübergehen erwähnt linden, so zun Beispiol der bekannte Fall von li. Bell, so waren doch der Mohrzahl der Aorzte jener Zeit, die hervorragendsten unter ihnen nicht abusgeschlossen, die Erscheinungen der Syphilis des Gehirns gänalich umbekannt. 
Der berühmte Hunter zum Beispiel zählte das Gehirn zu den dem Einflusse der Syphilis am wenigsten unterworfenen Organen.

Astley Cooper ging noch weiter und hielt die Gehirnsubstanz für unfähig, sich unter Einfluss der Syphilis za verändern.

Sogar im Anfange, selbst gegen die Mitte dieses Jahrbunderts wurde der Syphilis bei Erkrankungen des Nervensystems noch eine sehr geringe Aufmerksamkeit geschenkt. Man braucht nur die besten Lehrbücher für Nervenkrankheiten der Vierziger und Fünfziger Jahre, zum Beispiel, das von Marsh all-Hall oder Romberg durchzusehen, um sich zu überzeugen, wie wenig in ihnen von der Hirnsyphilis die Rede ist.

Ja noch später sprechen sich so hervorragende Geister und berühmte Fachmännor für Nervenkrankheiten, wie zum Beispiel Prof. Lasègue, über die Affectionen des Nervensystems unter Einfluss der Syphilis dahin aus, dass diese sehr selten vorkommen und ihren Erscheinungen wach wenig charakteristisch sind.

In den Sechziger Jahren hat Vir chow zuorst für die Bearbeitung der Syphilis des Nervensystems eine solide pathologiseh-anatomische Grundlage geschaffen. Wie Thnen bekaunt, hat er mit der ihm eigenen Präcision und Vollständigkeit die späten syphilitischen Affectionen der Schädelknochen, der Hirnhäute und der Hirnsubstanz selbst beschrieben.

Der Vielfältigkeit der anatomischen Veränderungen entsprechend, trat dann anch die Klinik mit einer Reihe von Beobachtungen auf. Es erschienen Veröffentlichungen zahlreicher Fälle von Syphilis des Gehirns, des Rückenmarks und einzelner Nerven, wie zum Beispiel die von Lagneau, Gros und Lancereaux, Zambakó u. A.

Doch hat Niemand das Studium der Hirnsyphilis so sehr befördert, wie Dr. Heubuer durch seine 1874 veröffentlichten Untersuchungen über syphilitische Erkrankungen der Hirngefässe. Die syphilitische Arteritis kann, wie Heubner bewiesen und sodann Baumgarten, Cornil, Lancereaux u. A. bestätigt haben, ein beliebiges Hirngefäss, das grösste wie das kleinste, befallen und dadurch die Ernährung, später auch die Function sowohl eines einzelnen Gehirncentrums als auch eines 
gánzen Gehirngebietes stören. Es gibt also keine Affection des centralen Nervensystems, we]che nicht durch eine syphilitische Gefässerkrankung hervorgerufen werden könnte.

Prof. Fournier gehört sodann das grosse Verdienst, alle mannigfaltigen Erscheinungen der Hirnsyphilis in ein geordnetes Ganze gruppirt zu haben. Indem er eine trockene, in praktischer Hinsicht ziellose Classjficirung der enorm grossen Casuistik, wie es seine Vorgänger gemäss der in Lebrbüchern für Nervenkrankheiten üblichen Schablone gethan hatten, vermied, gelang es ibm, sich in dem angehäuften Material zurecht zu finden und in höchst talentroller Weise die einzelnen Typen der Hirnsyphilis hervorzuheben, wodurch der klinischen Beobachtung der richtige Weg gewiesen wurde.

Seine im Jahre 1879 erschienenen Vorlesungen: „La syphilis du cerveau" sind in der praktischen Medicin epochemachend gewesen. Seine weiteren Untersuchungen: "Do l'ataxie locomotrice d'originesyphilitique", im Jahre 1882 erschienen, und sein im Jahre 188: veröfentlichtes Werk: .. Legons sur la périodo préataxique du tabes l'origine syphilitique" laben am meisten dem allzu grossen Uebertreiben der Rolle der Syphilis in der Aetiologio dor Nervenkrankheiten Vorsehub geleistet.

Tu dieser Ueberschatzung haben in bedentendem Grade auch noch spätere Forscher mitgeholfen, zum Beispiel Dr. Mauriae, welcher bewiesen hat, dass nicht nur späte Formen der Syphilis sich im Gehirne localisiren können, sonderu dass auch die frühe, secundäre oder condylomatöse Syphilis dieses Organ befallen könne. Im 2., 3. Monate nach Beginn der Syphilis können sich Symptome Seitens des Gehirns entwickeln.

Ist also bei oinem Kranken Syphilis vorhanden, sei es eine siau frische, kaum entwickelte oder eine vor 20, 30 Jahren abgelaufene, so können die verschiedensten vorhandonen nervösen Störungen anf diese Ursache bezogen werden. Mit anderen Worten, alle nervösen Erscheinungen mit Syphilis in der Anamnese können Aeusserungen der Syphilis sein. Die klinische Diaguose und Therepio nervöser Erscheinungon bei Syphilitischen ist dadureh in hohem Gride vereinficht worden. 
Finden wir bei einem Kranken mit Affection des centralen Nervensystems Syphilis in der Anamnese, oder sind gar gleichzeitig lnetische Erscheinungen vorhanden, so müssen wir ihm die mercurielle oder besser eine gemischte Behandlung mit Jod und Mercur verschroiben. Einfach und klar. Hauptsächlich einfach.

Auch die erhaltenen praktischen Resultate waren anscheinend gute.

Schwanden die Erscheinungen, so war das ein Beweis, dass sie in der That syphilitische gewesen waren. Gingen die Erscheinungen nicht zurück, nun so war die Behandlung nicht energisch genug oder zu spät begonnen worden. Fs muss also unter dem Einfiusse der Syphilis zu stationären Bildungen in den Hirnhäuten und Gefässen gekommen sein; es muss sich compactes Bindegewebe gebildet, Obliteration in den Gefässen eingestellt haben, und die Function des vom afficirten Gefässe ernährten Gebietes des Gehirns lässt sich nicht mehr wiederherstellen. Dio Flagge der pathologischen Anatomie schütate in dieser Weise jede therapentische Ladung, gute sowohl als schlechte.

Allmälig nahm diese in wissenschaftlicher Hinsicht vereinfachte Diagnosestellung den Charakter eines gerichtlichen Verhörs an, in dem der Kranke die Rolle des Angeklagten spielte und rom Arzte als Untersuchungsrichter aufs Strengste, nicht selten mit leidenschaftlicher Voreingenommenheit befragt wurde, ob er nicht jemals etwas dem Schanker, der Syphilis oder einer venerischen Krankheit Aehnliches gehabt habe. Jeder Zweifel Seitens des Angeklagten wurde zu Gunsten des Untersuchungsrichters ausgelegt und als Resultat kleiner Ungenauigkeiten in den Angaben, Vergessen und Missverständnissen orgab sich die Diagnose, wie ein auf Kanzleiacten beruhender Gerichtsspruch aus alter Zeit, mit Ausschluss der modernen Rechtsmittel.

Doch ist diese glückliche Zeit der Verhörsdiagnose - für mich wenigstens - unwiederbringlich vorüber. Mein Glaube an die diagnostische Kraft der Anamnese ist verschwunden, ich habe aufgebört im Mercur und Jod sichere und zuverlässige Heilmittel aller nervösen Leiden der Syphilitischen zu sehen und glaube selbst an die untrügliche Bedeutung der Coincidenz der Erscheinungen nicht mehr, 
Diese Enttäuschung in der Tauglichkeit des gerichtlichen Verhörs für die Diagnose der Hirnsyphilis ist es, worüber ich mich hier vor Ihnen in Kürze anssprechen möchte.

In der Geschichte der Syphilis haben sich solche Episoden „erleichterter Diagnose“ anf Grund der Anamnese, begleitender Erscheinungen und der Probebehandlung nicht ein Mal wiederholt. Und nicht ein Mal erwies sich die bei geringem Wissen einfache Diagnose als sehr complicirt, wenn die Kenntnisse sich erweiterten. In dieser Hinsicht ist dio klinische Geschichte der Syphilis höchst lehrreich.

Vor kaum 50 Jahren waren unsere Kenntnisse über Erkrankungen dor Haut äborhaupt nur sehr mässigen Umfings; ebenso waren auch die Syphilide, sowohl von pathologischanatomischer, als auch klinischer Scito in gonügender Weise noch njeht bokannt. Damals waren die Beriohungen der Hantsyphilis zu anteren, niwht specifischon lirklankungen dor Hautbedeckung ganz die nämlichen, wie sie jetzt zwischen der Syphilis des Norven-

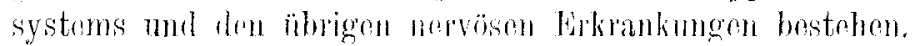

Iamals stïtate sich dio Diagnose irgent aimer syphilitischon

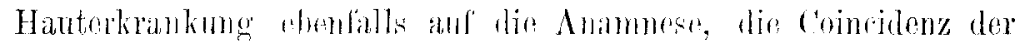
Erscheinungen und dia Probohndandhung. So entstand dio Psoriasis syphilitica, der Lapus syphiliticus, der Pemphigus syphiliticus, das Fezema syphiliticum und selbst dio Seabies syphilitica! Kurz, es gab koine vulgäre Hanterkrankung, die nicht eine entsprechende Form der Hautsyphilis besass. Ganz ebenso haben wir jetzt eine syphilitische Epilepsie, eine syphilitische Verrücktheit, eine syphilitische Tabes und selbst eine syphilitische Hysterie.

Wie es damals möglich war, ohne sich in das Gebiet der Dermatologie zu vertiefen, leichten Herzens und mit schwachen Kenntnissen die Diaguose auf syphilitische Hautaffectionen zu stellen, so ist scheinbar auch jetzt kein besonderes Studium der Nervenkrankheiten nöthig, um die Entscheidung und besonders die passende Therapie bei einem beliebigen nervösen Leiden eines Menschen zu treffen, der die Syphilis gehabt hat. War ein Schanker vorhanden, hat der Kranke Halsschmerzen gehabt, waren ihm die Harare ausgefallen - das genügt vollkommen: der vorhandene Ausschlag muss ein syphilitischer sein; geben Sie oder versuchen Sie ihm jedenfalls Mercur und Jod zu geben, Fand 
man dazu noch neben dem Ausschlage irgend welche syphilitische Affectionen der Schleimhäute, Muskeln, Knochen oder der Knorpel, so durfte durchans kein Zwejfel daran entstehen, dass die einzige Rettung des Kranken ausschliesslich in einer specifischen Behandlung bestehen könne.

So sprach man vor 50 Jahren, wenn man an die Diagnose und Therapie von Erkrankungen der Hautbedeckung bei Syphilitikern ging; ganz ebenso urtheilt man jetzt bei der Diagnose der Hirnsyphilis.

Für die Syphilide nun ist die Zeit der „,rleichterten Diagnose" für immer vorüber. Jetzt weiss es ein jeder Arzt, dass man ohne vollständige Kenntniss der Dermatologie auch die Hautsyphilis nicht verstehen kann. Ebenso wird kein moderner Arzt eine Hautaffection nur deshalb für ein Syphilid halten, weil sie sich bei einem Menschen entwickelt hat, welcher Syphilis gehabt hat oder noch hat, oder weil sie einer mercuriellen oder Jodbehandlung gewichen ist. So hält z. B. jetzt Niemand die Psoriasis vulgaris für eine Erscheinung der Syphilis auf Grund dessen, dass diese Form oft bei Menschen beobachtet wird, welche Syphilis überstanden haben oder gar noch syphilitische Erscheinungen darbieten, oder deshalb, weil dieser Ausschlag unter Einfluss grosser Dosen Jodkali vergeht. Die Diagnose der Syphilide stützt sich jetzt auf die objectiven Anzeichen, welche dieselben hinsichtlich ihrer Structur, Verlauf, Localisation, ihrer Ausgänge darbieten. Die Anamnese und die begleitenden Erscheinungen, ebenso wie die Probebehandlung spielen jetzt in besagter Diagnose gar keine Rolle mehr.

Jetzt sehe ich im Laufe eines jeden Lebrjahres mit grossem Vergnügen, wie die Studenten der höheren Curse, obne den Kranken zu befragen, die Syphilis der Hautbedeckung mit Bestimmtheit diagnosticiren und etwaige gleichzeitig vorhandene, nicht specifische Hautaffectionen dabei unterscheiden.

Ich wiederhole, mit der Syphilis des Nervensystems steht es bei uns jetzt ebenso, wie es vor 50 Jahren mit der Hautsyphilis stand.

Ob der Kranke Paralyse oder Convulsionen hat, ob Neuralgien oder Anästhesien, ob Verrücktheit oder Aphasie - ist gleichgiltig; nicht die Erscheinungen sind wichtig, sondern die Frage: 
Hat or Syphilis gehabt oder nicht? Wenn er sie gehabt hat, ob auch vor 20, 30 Jahren, - geben Sie ihm Mercur und Jod, geben Sie es energisch und erwarten Sie Erfolg; tritt dieser nicht ein, so kann Ihr Gewissen dennoch rubig sein - Sio haben gethan, was möglich ist - der Kranke ging lege artis zu Grunde.

Das Uebertreiben der Rolle der Syphilis in der Aetiologie nervöser Leiden hat Fanatiker dieser Idee geschaffen. Zu diesen gehört z. B. Prof. Erb, welcher einen Fall von Tabes nicht nur dann für unzweifelhatt syphilitischen Ursprungs erklärt, wenn er sich überzengt hat, dass der Kranke in der That an Syphilis gelitten, sondern anch damn, wonn or erfïhrt, dass der Kranke ein Mal einon Schanker gohabt hat, ja solbst wem or nur eine Narbe nach einem Geschwür an den Genitalien vorfindet. Oder \%. B. Prof. Kjellberg (Upsalia), für den eine progressive Paralyse ohne Syphilis überhaupt nieht existirt. Wemn der Kranko keine acruirirte Syphilis, wenn er selbst nicht den geringsten Schanker oder Gonorthöe gehaht hat, so hat das in den Angen von Prof. Kjollborg Nichts zu bodenten. Der Kranke muss eine hereditäre Syphilis habon vom Vater oler von dar Mntter, vielleieht sogar vom Grossvater ofor der Grosemutter.

Dio Voreingenommenheit für die Syphilis als Letiologio für die Frkankungen des Nervensystems hat anscheinend eino Cxundejgensehaft dieser Krankhoit vergessen lassen, dass sio nämljch koinen einzigon pathologischon Procoss aussehliesst. Ebenso wio alle uns bokanuten nicht specifischeu Hanterkrankungen sich anch bei Syphilitischen einstellen können, ohne die ihnen charakteristischen Merkmale zu verlieren, ganz ebenso können sich alle möglichen Affectionen des Nervensystems bei einem an Syphilis leidenden Menschen entwickeln.

Schon Das allein genügt, um Zweifel an der Richtigkeit einer Diagnose aufkommen zu lassen, die auf dem alten, aber nicht richtigen Satze - post hoc ergo propter hoc - beruht nach der Syphilis, also in Folge von Syphilis.

In der That, je grössere Fortschritte das Studium der Nervenkrankheiten besonders in Frankreich und England in den letzten Jahren gemacht hat, je mehr die Neuropathologen durch Bearbeitung der Physiologie, pathologischen Anatomie und Klinik das Chaos der nervösen Symptome vermittelst genauer Kenntnisse 
geklärt und sie den pathologischen Processen gemäss in Krankheitstypen geordnet haben, desto prägnanter trat die Mangelhaftigkeit der auf der Anamnese und den begleitenden Erscheinungen, d. h. rein zufälligem Zusammenfallen von Krankheitssymptomen begründeten Diagnose hervor. Noin, nicht auf den Zufall soll sich jetzt eine wissenschaftliche klinische Diagnose stützen, sondern auf das Studium der nervösen Erscheinungen selbst, ihrer Entwickelung, Besonderheiten ihres Verlaufes, gegenseitigen Zusammenhang, charakteristische Gruppirung, Verschiedenheiten der Ausgänge, der Complicationen; ondlich auf die Summe aller krankhaften $\mathrm{Ab}$ weichungen des betreffenden Organismus.

Auf diesen Weg tritt gegenwärtig die Syphilidologie: Es ist zwar ein schwerer Weg, welcher es einem jeden Syphilidologen zur Pflicht macht, die nervösen Krankheiten näher kennen zu lernen, dafür aber anch der einzige, welcher die Möglichkeit gibt, bei der Diagnose und Therapie nervöser Erscheinungen bei Syphilitikern mit vollem Bewusstsein zu handeln.

Jetzt genügt es nicht mehr zu sagen: der Kranke hat Syphilis gehabt und hat jetzt nervöse Erscheinungen - geben Sie ihm Mercur und Jod. Neiu, jetzt heisst es: der Kranke hat die Syphilis und ausserdem nervöse Erscheinungen; es gilt zu entscheiden, welcher Art diese Erscheinungen sind. Wenn diese nervösen Erscheinungen der Hysterie, Neurasthenie, Tabes, Paralysis progressiva oder einem anderen nicht specifischen pathologischen Processe angehören, so können wir dem Kranken durch Verordnung von Mereur schaden. Sind aber die betreffenden nervösen Erscheinungen wirklich der Ausdruck einer Hirnsyphilis, dann und nur dann müssen wir zur specifischen Behandlung greifen, welche in diesem Falle fast immer Heilung erzielt oder wenigstens dem Kranken grossen Nutzen und keinen Sehaden bringt.

Aber, werden mir die Anhänger der alten Ordnung erwiderv, ist denn eine Möglichkeit vorhanden, auf Grund irgend eines Symptomes seitens des centralen Nervensystems die Ursache des uns vorliegenden Leidens zu erkennen? Ist es beispielsweise möglich, bei Lähmung irgend einer Muskelgruppe zu eruiren, ob diese Lähmung durch ein Gumma im Gehirne oder durch irgend einen anderen 
mit der Syphilis nichts gemein habenden malignen Tumor oder eine Apoplexie im Gehirn bedingt sei?

Freilich lässt sich auf Grund eines einzeln genommenen nervösen Symptomes die dasselbe bedingende Ursache nicht bestimmen; wie es nicht möglich ist, auf Grund nur eines mikroskopischen Präparates Veränderungen des Gehirns, die durch Syphilis hervorgerufen sind, von denjenigen der progressiven Paralyse z. B. zu unterscheiden. Doch verfolgen Sie die betreffende Erscheinung, studiren Sie ihre Entwickelung und ihren Verlauf, bringen Sie dieselbe mit anderen bei diesem Kranken zur Beobachtung kommenden nervösen Symptomen in Zusammenhang, und Sie werden gewiss in der grossen Mehrzahl der Fälle im Stande sein, unabhängig von der Anamnese, mit grosser Bestimmtheit den zu Grunde liegenden pathologischen Procoss zu erkennen.

Der schon länst von Buzard aufgestellte Vergleich dos erkrankten Gehirns mit giner verdorbenen Uhr, wolchon alle Jiobbaber des Verhörswesens in der Diagnose so gern eitiren, ist nicht stichhaltig, oder richtiger, er trifft nicht das Wesen der Sache. Ob ein Sandkosn oder ein Märehon den Uhrmechanismus zum Stehen gebracht hat, sagt man, --- in beiden Fällen bleibe die resultirende Störung die nämliche, unabhängig von der bewirkenden Ursache. Dasselbe sehen wir im Gehirn: ob das Nervencentrum oder ein Hirngefäss durch ein Gumma, eine carcinomatöse Neubildung oder einen 'Tuberkel Druck erfährt - die entstandenen Störungen bleihen dieselben, unabhängig von den Figenschaften des Tumors.

Dieser Vergleich ist hübsch, aber leider nicht richtig. In der That wird ein Sandkorn und ein Härchen den Gang der Uhr in gleicher Weise stören und Niemand wird im Stande sein, die Ursache zu nennen, ohne sie gesehen zu haben. Doch geben Sie die Uhr einem erfahrenen Meister und dieser wird nach aufmerksamer Besichtigung, ohne den ganzen Mechanismus auseinanderzunehmen, ohne aachzufragen, sofort erkennen, ob hier ein namhafter Bruch oder eine unbedentende Vermmreinigung durch verdicktes Oel, Stanb u. dgl. vorliegt.

Das Nämliche gilt vom Gehirn: ein erfahrener und wissensreicher Meister kann und muss durch genaue Untersuchung, ohne den ganzen Mechanismus durch die Section auseinanderzulegen, 
ermitteln können, welche Ursache die betreffende Störung bewirkt hat. Dass nun die Diagnose der centralen nervösen Affectionen wirklich in dieser Richtung sich entfaltet, das unterliegt durchaus keinem Zweifel.

Vor kaum 12 Jahren sagte Prof. Fournier in seinen Vorlesungen über die Hirnsyphilis, dass selbst der erfahrenste und an Kenntnissen reichste Arzt einen epileptischen Anfall beobachtend, nicht im Stande wäre zu entscheiden, ob dieser Anfall durch die Localisation der Syphilis in der Hirnrinde bedingt. sei oder als Ausdruck einer essentiellen, mit der Syphilis Nichts gemein habenden Epilepsie auftrete.

Was sehen wir aber gegenwärtig?

Während dieser 13 Jahre ist unsere klinische Erfahrung soweit gewachsen, die Besonderheiten verschiedener epileptoider Zustände sind soweit eruirt worden, dass jeder moderne Arzt in der grossen Mehrzahl der Fälle auf Grund der Beobachtung eines epileptoiden Anfalles mit Gewissheit sagen wird: dieser Anfall erscheint als Ausdruck einer Hystero-Epilepsie, deron Bild von Prof. Charcot in so prägnanter Weise gezeichnet worden ist; jener, ein partieller oder mit Erhaltung des Bewusstseins einhergehender, ist eine Jackson'sche Epilepsie, welche grösstentheils als Erscheinung der Syphilis auftritt; jener dritte Kranke bietet den Typus der rein essentiellen Epilepsie (malum comitiale), welche so lange jeder Bohandlung trotzt. Endlich wird der Arzt bei Untersuchung eines vierten Kranken sagen, dass bei ihm der epileptoide Anfall den Beginn einer unheilbaren, tödtlichen Krankheit - der progressiven Paralyse andentet. Und um das Alles zu entscheiden, ist es gar nicht nothwendig, den Kranken einem Verhöre zu unterwerfen - man muss nur verstehen zu beobachten.

Und nicht die convulsiven Anfälle allein enthalten in sich selbst - in der Summe aller sie zusammensetzenden, sowohl vorhergehenden, als auch nachfolgenden Erscheinungen - die Merkmale, welche uns die Möglichkeit geben, ohne den Mechanismus zu zerlegen, mit Bestimmtheit die den Anfall bedingende Ursache zu ermitteln.

Betrachten wir die Lähmungen. Bei einem jungen Menschen, der an Syphilis gelitten, sehen wir Lähmung oder Parese der 
Augenmuskeln - ja das ist zweifellos eine nervöse Erscheinung syphilitischen Ursprungs! Das wird die Meinung der Mehrzahl der praktischen Aerzte der Jetztzeit sein.

Geben Sie ihm ohne Weiteres Mercur _- es wird helfen. Ja, es hilft auch in der That, aber wie? In einem Falle wird die Lähmung verschwinden und sogar recht schnell - in 2, 3 Wochen, und nichtsdestoweniger wird dieser Fall für den Kranken ein schwerer sein. Die Paralyse wird vergehen, der Arzt wird triumphiren und noch mehr in der Richtigkeit seiner leichten, raschen und genauen, auf die Anamnese begründeten Diagnose bestärkt werden. Doch werden die den Kranken anfallsweise beunruhigenden rheumatalgischen Schunorzen nicht vergehen, oder sogar olne sichtbaren Grund zunehmon; der Kranke bemerkt, dass ihm das Urinireu erschwert ist, sodann werden die Erectionen schwächor, allmälig treten Limpfindungen von Vertaubung an verschiedenen Partien des Körpers auf, taumelnder Gang, und es entwickelt sich endlich nach einer Reihe von Jalıren langsam, doch unaufhaltsam, das volle Bild der Tabes, obwohl wiederholt eine energische specifische Behandlung eingeleitet worden war.

In einem anderen Fallo dagegen wird die Augenmuskellähmung vielleicht schworer nachgeben, vielloicht oinige Monate zo ihrer Heilung arfordern. Eimmal geheilt, wird sie aber die lintwickolung der Tabes nicht nach sich führen. Diese Paralyso wird in der 'That eine tirscheinung' der Syphilis gewesen sein, wolche an der Hirnbasis localisirt war, eine Erscheinung, mit wolcher nicht selten das Bild der Hirnsyphilis beginnt.

Weiter, man wird noch eine keihe von Augon muskellähmungen beobahten, denon obenfibls syphilis volangegangen war, bei denen Mercur und Jod Anlangs schoinbar Nutzen bringen werden, bei denen aber, der Bohandlung ungeachtot, inmor ohne Ausnahme der 'lod eintreton wird. Iel meine die progressive Paralyso.

In allen solehen hällen muss man sich zurochtfinden können, unabhängig davon, ob der Kranke an Syphilis gelitten hat oder nicht, denu bei allen erwähnten Kategorien von Beobachtungen findet man Syphilis in der Anamnese. 
In unserer Zeit ist es nicht schwer, auf Grund der Untersuchung in jedem einzelnen Falle mit Gewissheit zu entscheiden, ob wir es mit einer syphilitischen, tabetischen oder paralytischen Augenaffection zu thun haben. Wir wissen, dass die einseitige Affection eines Astes des N. oculomotorius, welche z. B. Ptosis bedingt hat, bei gleichzeitigem Vorhandensein des Symptomes von Argyll-Robertson mit Bestimmtheit darauf hinweist, dass wir es mit der tabetisehen Form zu thun haben; wir wissen ebenso, dass eine Myosis spinalis, welche, sagen wir, die Parese des M. rectus externus begloitet, ebenfalls zu Gunsten der Tabes spricht. Während dagegen eine Diplopie, z. B. durch Affection verschiedener die Augenmuskeln innervirenden Nervenäste an beiden Seiten bedingt, immer für die syphilitische Natur des Leidens spricht.

Wir wissen andererseits, dass eine Mydriasis, welche unbemerkt ohne irgend welche andere Veränderungen des Muskelapparates des Auges eingetreten, aber von Zittern der Lippen, der Zunge, Sprachstörung, Veränderungen der Psychik begleitet ist, dem Kranken mit Untergang unter Erscheinungen dos paralytischen Blödsinnes droht.

Nicht die Augenmuskellähmungen allein sind es, welche bei genaner Untersuchung ohne Befragen des Kranken in einem Falle auf don zweifellos syphilitischen Ursprung des Leidens hinweisen und die Möglichkeit einer sicheren Heilung in Aussicht stellen, in einem anderen Falle dagegen die Einleitung der specifischen Behandlung verbieten.

Nicht die Lähmungen allein, sage ich, soudern noch viele andere klinisch genau bearbeitete, prägnant markirte Symptomencomplexe geben, vom neuropathologischen Standpunkte betrachtet, zweifellose Hinweise auf die sie bewirkenden Ursachen, lassen den krankhaften Vorgang, als dessen Ausdruck sie auftreten, erkennen.

Wer von den Neuropathologen wird jetzt einzig und allein auf Grund genauer Beobachtung des Zitterns der Glieder eines Kranken nicht fehlerlos entscheiden, ob er es mit chronischer Quecksilbervergiftung, Alkoholismus, progressiver Paralyse, Hysterie zu thun hat, oder ob der Kranke an Paralysis agitans, disseminirter Sklerose oller senilem Zittern leidet? 
Wer wird nicht nach der Aussprache oder der Schrift des Kranken einen Hemiplegiker von einem progressiven Paralytiker oder Letzteren von einem an disseminirter Hirnsklerose Leidenden unterscheiden konnen?

Von Tag zu Tag wird uns mit der wachsenden Bereicherung unseres Wissens mehr und mehr die Möglichkeit gegeben, die einzelnen pathologischen Processe im Gehirn genauer und frühzeitiger zu erkennen und zu differenziren. Diesem eingehenden klinischen, durch pathologisch-anatomische Untersuchungen controlirten Studium verdanken wir es jetzt, dass sich von der Syphilis des Nervensystems zwei umfangreiche und complicirte 'Typen, nämlich die Tabes und die progressive Paralyse abgetrennt haben, worauf ich gerade Ihre Aufmerksamkeit lenken möchte.

Trotz positiver Hinweise der Anatomo-Pathologen auf den degenerativen Charakter der Veränderungen bei Tabes, die diffuse Meningo-Encephalitis bei progressiver Paralyse und ausgeprägten Herdaffectionen bei der Syphilis, trotz dieser wesentlichen Unterschiede der erwähnten pathologischen Processe war die Uebersebätzung der Rolle dor Syphilis als ätiologisches Moment verschiedener nervöser Erscheinungen so gross, dass noch jetzt sohr viele Aerzte os für ihre unumgängliche Pflicht lialten, die progressive Paralyse und die Tahes specifisch zu behandeln, und uur deshalb, woil diese beiden Krankheiten grösstenthais bei Leuten vorkommon, wolche syphilis gehabt haben.

Ieh gohe noch woiter, ich bin übormongt, dass sich unter meiuon Zuhörern mohr als ein Arzt findon wird. welcher anf Grund seiner Beobachtungen innig iblerzeugt ist, dass er durch Meren. progressive Paralyse oder Tabos bei Syphilitischen geheilt - wenn auch nicht ganz geheilt hat, so doch dem Kranken in bedeutendem Grade geholfen habe.

leh glanbe dem sehr goin, indem ieh vor 12 bis 15 Jahren derselben Ansicht huldigte, und - ich beeilo mich das hinzuzufügen - die Ueberzengung, dass der Tabes und progressiven Paralyse kein syphilitischer Charakter zuzusprechen ist, hat mich viel Zoit, Mühe und nicht wenig Zwoifel, Misserfolge und Versehen gekostet.

Vor Allem werde ich an der Coincidenz der erwähnten krankhaften Vorgänge mit der Syphilis stehen bleiben. 
Ja, die Tabes und progressive Paralyse kommen bei Leuten, die an Syphilis gelitten haben, häufiger vor, als bei solchen, die diese Krankheit nicht gehabt haben. Beweist dies aber, dass die erwähnten Processe eine Erscheinung der Syphilis darbieten? Durchaus nicht, denn sie kommen, wenn auch seltener, doch zweifellos unabhängig von Syphilis vor und bewahren dabei alle ihre anatomischen und klinischen Besonderheiten.

Nehmen wir ein anderes mehr demonstratives Beispiel ähnlichen Zusammenfallens; freilich ist dieses nicht rein zufällig, sondern steht in einem gewissen causalen Zusammenhange, von dem weiter unten die Rede sein wird.

Sie wissen, dass Keloidbildungen (Narbenkeloide) sich an Narben nach Syphiliden am hänfigsten entwickeln. Bilden sie eine Erscheinung der Syphilis? Niemals, denn sie kommen, wenn auch viel seltener, beispielsweise an Narben nach Brandwunden vor und haben dabei den nämlichen anatomischen und klinischen Charakter. Sammeln Sie aber die statistischen Daten, da finden Sie von 100 Keloiden bei 90 Syphilis in der Anamnese, ebenso wie es nach Beobachtung von Erb und Fournier bei der Tabes und Paralysis progressiva der Fall ist. Viele Aerzte (Wilck, Thomas Briand u. A.) haben auf Grund dessen die Keloidnarben für Erscheinungen der Syphilis gebalten und sie eifrig mit Mercur oder Jod behandelt.

Indessen muss man die schöne, von einem berühmten Statistiker ausgesprochene Regel nicht vergessen: „la statistique trouve, mais ne prouve jamais". - In der That erweist die Statistik die Gesetzmässigkeit einer gewissen Art von Erscheinungen, ohne ihre Causalität zn erklären. Daher haben sich alle objectiven Beobachter trotz aller statistischen Beweise bald davon überzeugt, dass, möge man den Kranken mit Quecksilber zu Tode curiren, die Keloidnarbe dennoch unverändert bleibt oder langsam immer weiter wuchert.

Andererseits weist dieselbe Statistik aul eine andere Reihe vou Thatsachen hin. Die Hirnsyphilis offenbart sich bekanntlich bei Männern und Woibern durch gleiche Erscheinungen. Sich im Gehirn localisirend, ruft sie bei beiden in gleicher Weise Lähmungen, convulsive Anfälle und Störungen der Psyche hervor, - Alles, ausser der Tabes und der progressiven Paralyso. Diese 
beiden Krankheitsformen, welche bei syphilitischen Mäınern eine alltägliche Erscheinung bilden, kommen bei an derselben Krankheit leidenden Frauen als eine seltene Ausuahme vor.

Woher kommt es nun, dass sich auf 50 männliche Tabetiker mit Syphilis in der Anamnese, kaum eine unter gleichen Bedingungen stehende Frau mit Tabes findet. Weshalb wird das Nämliche auch hinsichtlich der progressiven Paralyse boobachtet?

Offenbar gibt es ausser der Syphilis noch viele, uns bisher unbekannte Ursachen, welche die Entwickelung der Tabes und der progressiven Paralyse sowohl boi Syphilis, als auch ohne dieselbe bedingen.

Leider ist es bei der progressiven Paralyse und labes sehr schwer, sich von der Nutzlosigkeit oder, richtiger, Schädlichkeit der mercuriellen Behandlung zu überzeugen. First in den letzten 10, 15 Jahron hat das klinische Studium der Tabes und der progressiven Paralyse, besonders ilırer Anfuggsstudion, grössere Fortschritte gemacht. Jotzt wissen wir zum Beispiel, dass die Paralysis progressiva Jange Zeit vor dem Auftreten von Veränderungen der psychischen Sphäre dureh eine Reihe nervöser Erscheinungen sich äussern kinu, wolcho bald spontan verschwinden, bald, nach mehr wenigor langen Zeitintervallen mit verhältnissmässig gesundem Zustindo von Nouem wieder orscheinen. Jetzt treffen die frühoren birwïgungon bezüglich der Unwirksankeit der specifischen behandlung bei den in livelo stehenden krankhaften Processen nicht mehr au, und ihre Unwirksankeit kamm nicht meh" durch eine zu spät eingoleitete Behandlung orkliat worlen. Noin: Sowohl dio progressive Paralyse als auch dio T'abes können iu ihren Initialstadien diagnosticirt werden. Und gerade im Beginno dieser pathologischen Procosse werden tomporilre Bessorungen und spontaner Stillstand dor Bntwickolung anf ganze Monate und Jahre beobichtot, wolcho inn meiston irrezulühren gooignot sind.

Dor Kribuke atequirirt dio Syphilis, libst sich behandeh dio Erscheinungen verschwinden. Nible 5 bis 6 Jahren trith cin apoplektiformer Aufiall mit nachfolgonder Homiparese oder ein epiloptoider Aulall mit Vorlust dos Bowusstseins ant. Die psychische sphäre bleibt anschoinend normal. Lis wird die merenrielle Behandlung verordnet, und in einigou Wochen wird der Kranke vollkommen gesund. We vergelt oin halbes, ein Jahr. wieder ein 
Anfall - wiederum die specifische Behandlung und wiederum Besserung. Der Kranke regulirt seine Lebensweise, vermeidet Aufregungen, Sorgen, Anstrengung, und die Besserung hält an. Sodann treten nene Symptome auf: Sprachbeschwerden zum Beispiel, wiederum die Behandlung und noch einmal tritt einige Besserung ein. Während man auf solche Weise dem Kranken in einzelnen, ausgeprägten Krankheitssymptomen Hilfe leistet, gelangt man zu einem maniakalischen Anfall oder zu ausgeprägter Demenz, wo es unmöglich wird, den Kranken zu Hause zu behalten; man bringt ibn deshalb in eine Irrenanstalt mit ausgesprochener progressiver Paralyse. Indessen vergehen aber von der ersten nervösen Erscheinung, z. B. der Parese eines Aestehens des Nervus oculomotorius oder dem epileptischen Anfall, durch den sich der Process zuerst offenbart, bis zur ausgesprochenen progressiven Paralyse oft 5 bis 6 , sogar 10 Jahre. Während dieses ganzen Zeitraumes werden die spontanen Besserungen und temporären Stillstände in der weiteren Entwickelung der Krankheit, indem sie mit der Verordnung specifischer Mittel zusammenfallen, die jeweiligen Beobachter vom Nutzen einer solchen Behandlung überzeugen. Gerade die zufälligen Beobachter, welche solche Kranke temporär während einer gewissen kurzen Zeitperiode behandeln, sind noch bisher von dem Nutzen der specifischen Quecksilberbehandlung der progressiven Paralyse Syphilitischer am meisten überzeugt.

Noch jetzt kann man in jedem beliebigen Curorte mit sehwefelhaltigen Quellen während einer Saison nicht einen, sondern gegen ein Dutzend von im Anfangsstadium der progressiven Paralyse befindlichen Kranken beobachten, welche, nachdem sie eine mercurielle Cur mit schwefelhältigen Bädern durchgemacht, den Curort anscheinend gebessert verlassen; doch nie führen diese Besserungen zur vollständigen Genesung, sondern stets bringen sie den Kranken ins Grab.

Wenigstens war es so in allen denjenigen Fällen von progressiver Paralyse bei Syphilitischen, welche ich Gelegenheit hatte, von Beginn der nervösen Erkrankung an während der 10 folgenden Jahre zu beobachten; in allen diesen Fällon, ohne $\theta$ ine einzige Ausnahme, hat die specifische Behandlung, wie frühzeitig sie immer auch eingeleitet wurde, nie Genesung zur Folge gehabt - 
die Krankheit endete stets mit dem 'Tode, und zwar um so schneller, jo mehr das Quecksilber missbraucht wurde.

Dabei muss ich bemerken, dass ich gewohnt bin, alle sich an mich wendenden Kranken genau zu notiren, und dass sich im Laufe meiner 30jährigen Praxis in Gemeinschaft mit den meiner Klinik entnommenen Beobachtungen ein beträchtliches Material angesammelt hat - ungefähr 800 Fälle verschiedener nervöser Leiden bei Syphilitischen. Bei der in diesem Jahre vorgenommenen Durchsicht erwähnter Beohachtungen bin ich zu dem endgiltigen Ergebnisse gekommen, dass es mir in dieser Zeit, zu meinem grössten Bedauern, njcht gelungen ist, auch nur einen einzigen Fall progressiver Paralyse, die sich bei einem evident Syphilitischen entwickelt hat, durch Quecksilber zu heilen.

Ich spreche natürlich von der echten progressiven Paralyso oder, richtiger, (lor diffusen Meningo-Encephalitis, nicht aber von demjenigen eigenartigen, zuweilen durch zufällige Localisation der Syphilis im Gehirm bedingten Symptomencomplexe, welcher theilweise deu Erscheinungen dor progressiven Paralyse ähnelt und von Fournier so talentvoll unter dem Namen ,Psendoparalysio progrossivo" beschrieben worden ist. Dieso Psoudoparalysis progrossiva, wolcho als pathologisch-anatomischer Process mit dor echten progressiven l'aralyse nichts gemein hat, weicht froilich der specitischen Behandlung in glinzender Woise and kann in volle Genesung äborgehen.

Allerdings ist ein Irrthum in dor Diagnose und eine Verwechselung der Psendoparalysis mit der echten immor möglich, besonders bei der jetzt vorherrschenden Richtung der Verhörsdiagnose aul' dom Gobieto der norvösen Erscheinungen bei Syphilitischon.

Andererseits habe ich, wem ich von der specifischen Behandlung spreche, ausschliesslich die Quecksilbercur im Auge, welche den Verlauf der progressiven Paralyse in schäulicher Weise beeinflusst; während hingegen das Jod, besonders dio Jodbrompräparate, welche bei vielen Erkrankungen des Nervensystems nichtsyphilitischen Charakters nutzbringend sind, auch für die symptomatische Behandlung der progressiven Paralyse mit Erfolg angewandt werden können. 
Noch schwieriger ist es, sich von der Nutzlosigkeit oder, richtiger, Schädlichkeit der specifischen Behandlung bei der Tabes der Syphilitischen zu überzengen. Schwieriger nicht nur aus dem Grunde, weil die Anfangserscheinungen der Tabes zuweilen spontan spurlos verschwinden und im Laufe der Krankheit spontane, nicht selten sehr lange anhaltende Besserungen eintreten, sondern auch noch deshalb, weil es Formen der Tabes gibt, die auf einer gewissen Höhe der Entwickelung gänzlich stehen bleiben oder so langsam fortsehreiten, dass der Kranke in Folge irgend einer intercurrenten Krankheit sterben kann, ehe er noch ans dor präataktischen Periode heraus ist. Wer kennt nicht den so oft von Prof. Charcot citirten Fall einer Frau, die 30 Jahre lang an - einer durch die Autopsio bestätigten Tabes gelitten hatte und im Alter von 70 Jahren an einer Lungenentzündung starb, indessen aber noch keine Erscheinungen der Ataxie darbot; also im präataktischen Stadium zu Grunde ging.

Ich sehe vollkommen ein, dass sehr Vieles zur Verordnung der specifischen Behandlung bei einem Tabetiker, welcher Syphilis gehabt hat, verleitet, ja sogar anspornt. Der Kranke hat an Syphilis gelitten; nach 2-3, zuweilen nach einem halben Jahre, in anderen Fällen nach 20 Jahren stellt sich z. B. Parese eines Augenmuskels ein. Man verordnet Mercur oder Jodkali - in 2, 3 Wochen ist Alles vorüber. Der Arzt sowohl, als auch der Kranke werden äberzeugt sein, dass die Erscheinungen unter dem Einfluss des Heilmittels verschwnulen sind. Besonders wird der Kranke eineu festen Glauben an die Wirksamkeit der Behandlung gewinnen, denn er wird es ja nicht wissen, dass wenn ihm der Arzt statt des Jods und Quecksilbers Elektricität, Hydrotherapie oder gar nichts verordnet hätte - das nämliche Resultat erzielt worden, d. h. die Parese in derselben Zeit verschwunden wäre.

Das betreffende Symptom ist zWar auf lange, zuweilen für immer verschwunden, d. h. wird nie mehr wiederkehren, der krankhafte Process aber, als dessen aufängliche Erscheinung dieses Symptom anftrat, geht ungehindert seinen weiteren Gang.

Nach und nach, zuweilen im Verlaufe mehrerer Jahre stellen sich lancinirende Schmerzen, Harnbeschwerden und Parästhesien 
der Finger ein, es tritt das Romberg'sche Symptom auf und es entrollt sich allmälig das volle Bild der präataktischen Periode. Dazu ist die Tabes in ihren Anfangserscheinungen so mannigfaltig, so oft prävaliren in dieser Periode Gebirn- und nicht Rückenmarkssymptome und zwar solche, die schnell der Behandlung weichen, resp. schnell ohne jede Behandlung vergehen, um durch andere, stabilere vertreten zu werden, dass nur eine sehr lange dauernde Beobachtung specifisch behandelter und nichtbehandelter Tabetiker die Grundlage zu wichtigen Schlussfolgerungen geben kann. Beobachtet man eine chronisch verlaufende Tabes bei Syphilitischen während 10, 15, 20 Jahren, so sieht man, wio die einmal aufgetretenen Erscheinungen des präataktischen Stadiums, bald besser werdend, bald sich verschlimmernd oder verändernd, beständig an Zahl und Intonsität wachsen und in einigen Fällen unaufhaltsam zur Entwickelung des vollen Bildes einer ausgesprochenon Tabos führon.

Andorerseits erweisen solche Jangjährige Beobachtungen am besten dio Nutzlosigkeit und solbst die positive Schüdlichkeit der merewiellen Behandlung bei Tahes.

Der Kranko macht mehrero Malo dio moncurielle Behandlung durch, dio firscheinuegen geben, bald temporï schwächer, bald intensiver werdend, im Resultat Versehlimmerung des Zustandes. Der Kranke vorwirt die specifische Bohandlungsweise. Argentum nitricum, Brom, Secale coruutum, Hydrotherapic, Massage, Elektricität u. A. m. - - Mles versucht der Unglückjiche, und nicht selten tritt nach einer dieser nichtspecifischen Heilmothoden eine stabile Besserung ein.

Die Krankheit bleibt auf einem gewissen Punkte der Entwickelung während 5, 6 Jahren, nachdem jede specifische Behandlung eingestellt worden ist, stehen, während sie unter Einfluss dieser fortwährend sich verschlimmerte und rasch vorwärts schritt.

Ich karn nicht umbin, ein Beispiel solcher Art vorzufübren. Zwei Brüder wurden in ein und demselben Jahre mit Syphilis inficirt. Beide wurden fast gleich lange Zeit mit Mercur und Jod behandelt. Beim älteren entwickelt sich 5 Jahre später Tabes, welche mit einem prägnanten Anfall von Paraplegie debutirte. Sogleich wird eine energische Inunctionseur verordnet. Die Para- 
plegie wird besser, doch entwickeln sich quälende lancinirende Schmerzen und Parästhesien. Der Kranke begibt sich in einen Curort mit schwefelhaltigen Quellen, wo er eine noch energischere mercurielle Behandlung durchmacht und kehrt mit dem ausgesprochenen Bilde der Ataxie zurück, welche ihn bis zu seinem Tode an den Krankenstuhl fesselt. Der ganze Verlauf währte $21 / 2$ Jahre unter ununterbrochener mercurieller und Jodbehandlung.

Beim anderen. Bruder offenbart sich die Tabes im 6. Jahre nach der Syphilisinfection durch vorhinderte Ejaculatio seninis bei Stunden lang dauernder Erection. Sodann verschwinden die Kniereflexe, es stellen sich Schmerzen beim Uriniren, Crises gastriques ein u. s. w. Der Kranke hat aus Furcht, das Schicksal seines Bruders zu theilen, seit Entwickelung der nervösen Erscheinungen nie Mercur gebrancht; er gebrauchte Brom, Argent. nitr., Nassage, Elektricität - und nun befindet er sich 8 Jahre lang im präataktischen Stadium, welches sehr langsam progressirt, und fühlt sich von Zeit zu Zeit vollkommen gesund.

Die schweren Fälle von Tabes sind für die Nutzlosigkeit der mercuriellen Behandlung am meisten beweisend. In dieser Hinsicht stimme ich Prof. Charcot bei, wonn er in seinen klinischen Vorlesungen sagt: "Sie finden, dass die Tabes eine Erscheinung der Syphilis sei? Gnt. Hier haben Sie eine eben erst beginnende Tabes, welche sich durch tabetische Atrophie des N. opticus an einer Seite documentirt. Der Process ist ganz im Initialstadium; behandeln Sie ihn mit Mercur - geben Sie dieses Mittel soviel und wie Sie können, nur bringen Sie den Process zum Stillstehen, beugen Sie dem Gesichtsverlust vor oder wenigstens verhindern Sie durch Ihre Behandlung, dass sich der nämliche Process am anderen Auge entwickle. Versuchen Sie, und Sie werden sich überzeugen, dass Sie dieses Ziel nie erreichen. Wieviel Mercur und Jod Sie auch immer diesem Kranken geben werden, er wird dennoch erblinden. Vorausgesetzt nur, dass die Atrophie des Sehnervs wirklich tabetischen Ursprungs ist." Das ist für eine grosse Mehrzahl von Fällon vollkommen richtig.

Ja noch mehr, bei Beobachtung vieler Tabetiker mit constant gewordenen Erscheinungen, bei welchen sich im Laufe der Tabes zweifellos syphilitische Erscheinungen an der Hant, den Knochen und Muskeln entwickelt hatten, habe ich unter Einfluss 
der mercuriellen Cur stets eine Verschlimmerung der tabetischen Erscheinungen, einen rascheren Verlauf des Processes bemerkt. Zudem habe ich nie einen wirklichen Stillstand in der Entwickelung des tabetischen Processes unter Einfluss einer noch so frühzeitig begonnenen, wiederholten mercuriellen Cur beobachtet; dagegen bahe ich ohne dieselbe mehrmals einen solchen Stillstand bei Tabetikern gesehen, in deren Anamnese die Syphilis vorhanden war.

Selbstverständlich spreche ich auch hier von der wahren Sklerose der Hinterstränge und nicht von der Pseudo-Tabes der Alkoboliston, wolche auch an syphilitischen Alkoholisten beobachtet wird und bei Abstinenz von Wein und bei Jodbehandlung leicht eine stationäre Bessermg ergobon kann.

Ansserdem kam anch die syphilitische Myelitis, wem sie zufallig dio Hinterstränge bofallt, in groben tougen das Bild der 'Tabos vortäuschen, doch ist sie bed aufmerksumer Untersuchung von der wahren Tabes leicht zu unterscheiden und geht natrirlich unter Einfluss der mercuriellen Behandlung in Gonesung oder doch bedeutende Besserung übur.

Auf Grund alles Gesagton, scheido ich die progressive Paralyse und die Tabes aus der Zahl der Manifestationen der Syphilis des Nervensystems gänzlich aus und kann jetzt behaupten: selue ich einen Syphilitiker mit nervösen Ėrscheinungen, bei welchem Tabes und progressive Paralyse ausgeschlossen werden können, so habe ich es von 100 Fällen in 90 am wahrscheinlichsten mit einer wintichen Hirnsyphilis zu thun, d. 1. mit einer in dor bei Weitsm grössten Mohrzahl der Falle heilbaren Erschoinung.

Ich erkläre mir die häufige Entwickelung der progressiven Paralyse und Tabes bei Syphilitischen durch den schwächenden Einfluss, welchen die Syphilis auf einen schon vordem zu gewissen patbologischen Processen des Nervensystems prädisponirten Organismus ausübt. Die Prädisposition zur Tabes und progressiven Paralyse besteht von der Geburt an und wird durch die Syphilis blos ans Tageslicht gebracht. Wären die eben erwähnten beiden Brüder nicht an Syphilis erkrankt, so würdo sich bei ihnen vielleicht auch die Tabes nicht entwickelt haben.

Hreilich kann die Prädisposition auch eine acquirirte sein; die Syphilis spielt daun in solchen Fällen die Rolle eines das 
Gefäss überfüllenden Tropfens. Wie dem auch sei, jedonfalls steht es fest, dass wenn die Syphilis einen Menschen befällt, der in keiner Weise zu Erkrankungen des Nervensystems prädisponirt ist, auch dann, wenn sie sich im Gehirne localisirt, grosse Hoffnung auf vollkommene Genesung rorhanden ist.

Ja, meine Herren, die Prognose der Hirnsyphilis ist durchaus nicht so absolut schlimm, wie Viele denken. Ich besitze nicht wenjge Beispiele, in denen die Hirnsyphilis, welche sich durch Aphasie, Hemiplegie, epileptoide Anfälle, ja protrahirte Anfälle von Amnesie geäussert hatte, nicht nur rasch und vollständig dem Mercur und Jod gewichen war, sondern dank einer consequenten wiederholten Behandlung dauernde Genesung für Jahrzehnte ergeben hat.

Vor mehr als 20 Jahren habe ich einen Fall syphilitischer Aphasie mit rechtsseitiger Hemiparese bei einem jungen Officier beschrieben. Jetzt babe ich nicht selten Gelegenheit, diesen Officier zu sehen; er ist vollkommen gesund und rersieht seinen Dienst.

Vor ungefähr 15 Jahren habe ich in Gemeinschaft mit Dr. Voss einen Kranken beobachtet, welcher vollständige lähmung der einen und Parese der anderen Körperhälfte, Amnesie, Aphasie und Erschwerung - richtiger Unmöglichkeit - des Schluckactes darbot. Wie ein Cadaver lag der Kranke während 2 Wochen mit einem halbgeöffneten Auge sprach- und bewegungslos. Eine energische Behandlung stellte ihn wieder her und hat eine dauernde, bis jetzt ohne Recidive anhaltende Heilung erzieit.

Vor etwa 6 Jahren behandelte ich in Gemeinschaft mit Dr. Tschetschott eine Kranke mit rechtsseitiger Hemiparese, bei welcher sich während 24 Stunden 16 Mal Anfälle partieller Epilepsie wiederholten; die Kranke kam während 8 Tagen fast gar nicht aus ibrem bewusstlosen Zustande heraus. Die mercurielle Behandlung schenkte ihr bis jetzt anhaltende Gesundheit. Solcher Beispiele könnte ich mehrere Dutzende anführen.

In der That, wenn es jemals dem Therapeuten vergönnt ist den Kranken im vollen Sinne des Wortes aus den Klauen des Todes zu befreien, so ist das in Fällen der Hirnsyphilis; doch nur wahrer Hirnsyphilis, nicht aber irgend eines anderen pathologischen, das Nervensystem eines Menschen, welcher Syphilis gehabt oder noch hat, afficirenden Processes. 
Leider sind vollkommen reine Fälle von Hirnsyphilis in dieser Hinsicht nicht so häufig, wie es auf den ersten Blick scheinen könnte. Darauf deutet unter Anderem die Aotiologie der uns interessirenden Localisation der Syphilis hin. Die Frage, was am häufigsten zur Localisation der Syphilis im Gehirn prädisponirt, kann theilweise auf Grund folgender Daten beantwortetwerden: Unter 100 Kranken mit unzweifelhafter Hirnsyphilis, bei denen ich eingehende und genaue anamnestische Angaben sammeln konnte, erwiesen sich 43 Procent mit chronischer Alkoholvergiftung, welche 2-25 Jahre der Syphilis vorangegangen war und grösstentheils auch während des Verlanfes der Syphilis mit kurzen Unterbrechungen fortbestand; 29 Procent waren Neurastheniker, von dewen bei 18 horeditäre Prädisposition zn nervösen Erkrankungen vorhanden war, woranf nervöse oder psychische Leiden der Eltern oder Blutsverwandten in aufsteigender linie hinwiesen. Bei 5 Kranken hatten aller Wahrscheinlichkeit, nach traumatische Verletzungen des Schädels, vor odor nach der Erkrankung an Syphilis, im Gehirn oinen Locus minoris resistentiae gesehaffen und dadurch die Localisation des krankhaften Processns daselhst bedingt. In if Fällen endlich orscheint wioderholto Veberanstrengung des Gehirns als am meisten hervortretendes Moment, welehes zur Affection des Gehims beigotragen hatte.') Und nur bei 23 Kraiken, welche bis dahin anseheinend vollkommon gesund gowesen waren und eine verhältnjssmässig normale Lebensweise geführt hatten, entwickelte sich später dio IIimsyphilis. Mier sogleich noch die Bemerkung, dass bei diesen 23 Kranken der Verlauf und Ausgang der Hirnsyphilis sich gïnstiger gestaltete, als bei den übrigen.

Andererseits ist es ja begreiflich, was für eine grosse Mannjofaltigkeit der Erscheinungen Kranke darbieten müssien, bei welchen z. B. in Folge hereditärer Belastung gleichzeitig mit den Erscheinungen der Hirnsyphilis, oder mit diesen abwechselnd, Symptome der Neurasthenie, Hysterie, Epilepsie, primären Verrücktheit anftraten oder zugleich mit der Hirnsyphilis eine chronische Alkoholvergiftung verlief.

Nicht nur nach der Syphilis könnou sich, wie wir oben ge-

1) Ohne genaue Angaben ïber die ärhnten 100 Kranken anzuführen, will ich hier nur bemerken, dass in 6 Fällen die hereditäre Prädisposition zu nervösen Erkrankungen mit Alkoholismus cornbinirt war. 
sehen, im Nervensystem mit der Syphilis nichts gemein habende $\mathrm{p}$ athologische Processe, wie $\mathrm{z}$. B. die Tabes und progressive Paralyse entwickeln, sondern im Laufe der Syphilis und gerade am häufigsten dann, wenn sio sich im Gehirno localisirt, können auch nervöse Erscheinungen ganz anderer Natur auftreten, welche nicht Aousserungen dieser Krankheit, sondern Folgen von hereditärer Belastung, Alkoholismus, Traumen u. s. w. sind.

Ein Kranker, der zweifellose Hirnsyphilis gehabt hat, kann mit primärer Verrücktheit enden oder an essentieller Epilepsie, Neurasthenie, Hysterie leiden.

Allerdings ist es etwas sehwieriger, sich in diesen verwickelten Symptomencomplexen zurechtzufinden, als beispielsweise ein neben Syphiliden aufgetretenes Eczem zu unterscheiden. Ja noch mehr, die klinische Analyse verschiedener pathologischer Processe des Nervensystems, welche bei einem Kranken, besonders bei einem Syphilitischen, gleichzeitjg verlanfen, bildet eines der schwersten und zugleich interessantesten Probleme der modernen praktischen Medicin. Doch gibt es dazu nur einen Weg. Wie vor 30, 40 Jahren die Diagnose der Syphilide durch das Studium der Hautkrankheiten feste Grundlagen erhielt, so gelangen wir jetat dazu, mit Hilfe gründlicher Kenntnisse aller, auch der von der Syphilis unabhängigen Erkrankungen des Nervensystems eine streng wissenschaftliche Diagnose der Erscheinungen der Hirnsyphilis zu stellen, jede im Laufe der Syphilis zur Beobachtung kommende nervöse Erscheinung richtig zu deuten, endlich eine richtige Prognose zu stellen und in gewissen Fällen mit voller Hoffnung auf Erfolg die Durchführung einer energischen specifischen Behandlung zu fordern.

Ich habe mir, meine Herren, deshalb erlaubt, so lange Ihre Aufmerksamkeit für die Nothwendigkeit einer engeren wissenschaftlichen Vereinigung der Syphilidologie und Neuropathologie in Anspruch zu nehmen, weil die Repräsentanten letzterwähnter Wissenschaft schnell vorwärts schreiten, und wir Syphilidologen keine Zeit verlieren dürfen, denn sonst riskiren wir in Sachen der Hirnsyphilis, nicht auf der Höhe unserer Specialität zu bleiben. Dass aber unsere Specialität blühe und in allen auf sie bezüglichen Fragen das entscheidende Wort besitze, das, glaube ich, wünschen Sie ebenso wie ich. 